\title{
ACMG 2016 Update on Noninvasive Prenatal Testing for Fetal Aneuploidy: Implications for India
}

\author{
I. C. Verma ${ }^{1} \cdot$ R. Dua-Puri ${ }^{1} \cdot$ S. Bijarnia-Mahay ${ }^{1}$
}

Received: 23 January 2017/ Accepted: 30 January 2017/Published online: 6 February 2017

(C) Society of Fetal Medicine 2017

\begin{abstract}
ACMG 2016 guidelines for noninvasive prenatal screening (NIPS) are examined in the Indian context, and recommendation made for their adoption. Currently in India, NIPS is not a test that can or should be offered directly to patients. Proper pre-test counseling is essential, during which, the different options should be explained so that the patients can choose the test most appropriate for their situation. NIPS has a greater detection rate than other screening methods (like biochemical screening or ultrasound markers of aneuploidy), and can be performed in place of conventional screening for Patau, Edwards, and Down syndromes, if the patient can afford the cost, and the ultrasound does not show a malformation that is not a part of aneuploidies. The NIPS should be performed at 12 weeks of gestation or later, to reduce 'no calls' due to low fetal fraction. In samples that contain less than $4 \%$ fetal fraction the confidence in the report is reduced. Testing for sex chromosomes can be included, as these abnormalities are associated with unpredictable prognosis (that is often burdensome), which proves unacceptable to most Indian couples. However, patients should be provided balanced information on the abnormality that has been detected and left to choose the course of action most suited to their situation. Screening for microdeletions can be done if the patient can afford the cost, as microdeletions are
\end{abstract}

\section{C. Verma}

jfm200@yahoo.co.in

R. Dua-Puri

ratnadpuri@yahoo.com

S. Bijarnia-Mahay

bijarnia@gmail.com

1 Institute of Medical Genetics and Genomics, Sir Ganga Ram Hospital, Rajender Nagar, New Delhi 110060, India more frequent than aneuploidies in young mothers. In some cases diagnosis of a microdeletion is helpful in management of the disorder in the neonatal period leading to a better outcome. The vendors should fulfill their responsibility of educating the patients before offering the test, and giving clear reports in simple understandable language. It is hoped that more vendors will perform the tests locally so that they become cheaper and affordable, as they have greater detection rate than other screening methods.

Keywords Noninvasive prenatal screening (NIPS) . Noninvasive prenatal testing (NIPT) - Cell-free fetal DNA . Fetal fraction · High-risk women - Low-risk women .

Trisomy $21 \cdot$ Trisomy $13 \cdot$ Aneuploidies $\cdot$ Sex chromosomal disorders $\cdot$ Microdeletions $\cdot$ Malignancies

Noninvasive prenatal screening for aneuploidies based on cell-free fetal DNA in maternal blood (NIPS) was introduced as a service in the USA in 2011 [1], and rapidly spread like wild fire, irreversibly changing prenatal screening, and markedly reducing the rate of invasive procedures such as chorionic villus sampling and amniocentesis [2]. Numerous associations issued guidelines for its use, but initially most of them recommended its use only in high-risk women [3-7]. As large number of studies extended its application to low-risk women, as well as going beyond aneuploidies to microdeletions and whole genome copy number variations, ACMG issued an update on this topic [8]. In this paper we examine the implications of the new recommendations for India. The NIP test was introduced in India in December 2012, [9], and has remained largely restricted to major cities. A large number of obstetricians, more especially from the peripheral centers and mofussil towns, are still ill informed about the test. 
The commercial companies are promoting the test in a big way, eyeing the huge market created by almost 26 million births every year in India, and every few months a new company forays into the market. It is imperative that obstetricians and fetal medicine specialists be aware of the new recommendations, so that the noninvasive prenatal test is used properly.

1. The updated statement points out that NIPS is a screening test, and not a diagnostic test. The committee therefore uses the acronym NIPS for this test, "s" standing for screening. (In this article we have used the words NIPS and NIPT interchangeably). There are two clear implications of this. As it is a screening test only, there remains a residual risk of disease, albeit very small, even if the test comes back normal; which means that a normal result on NIPS does not completely exclude the presence of aneuploidy. This must be kept in mind and also be explained to the patient. The residual risk is however much smaller than for other screening modalities, such as biochemical screening or ultrasound studies. In one large study [10] the residual risk for aneuploidies, or false negative rate was $9 / 11,103$ i.e. $0.008 \%$.

2. During the course of counseling pregnant women in India regarding NIPS, it is frequently observed that they desire a 100\% exclusion of abnormality. We take efforts to explain that no test can be $100 \%$ accurate, as the gold standard tests such as amniocentesis and chorionic villi sampling can also occasionally err in providing a prenatal diagnosis of chromosomal disease $[11,12]$. We inform that NIPS is a high efficiency screening test, based on the meta-analysis of 37 relevant studies [13], that showed a sensitivity and false positive rate of 99.2 and $0.09 \%$ for trisomy $21,96.3$ and $0.18 \%$ for trisomy $18,91.0$ and $0.13 \%$ for trisomy $13,90.3$ and $0.23 \%$ for monosomy $\mathrm{X}$, and 93 and $0.14 \%$ for the sex chromosomal abnormalities.

3. Another important implication of NIPS being a screening test is that in the event of a positive NIPS test, the diagnosis needs to be confirmed by an invasive procedure such as chorionic villus sampling or amniocentesis. False positives are more likely for sex chromosomal disorders rather than trisomy of chromosomes 21,18 or 13 . We must emphasize that in the event of a high risk for Down syndrome, aneuploidy of other chromosomes, or other cytogenetic abnormalities on NIPS, the pregnancy should never be terminated, but a diagnostic test should be performed instead. Some vendors provide a free amniocentesis if the NIPS comes out to be positive, and it is hoped that all vendors in India should provide this option without charge.
4. The update also emphasizes that the patient be clearly told what NIPS does not provide. NIPS cannot be used to detect all chromosomal abnormalities, missing out on abnormalities of chromosomes other than $21,18,13$ and sex chromosomes, as well as structural abnormalities and translocations. It does not diagnose congenital malformations like spina bifida or congenital heart disease, it cannot predict abnormal pregnancy outcomes like growth restriction or preeclampsia, and it does not, at present, test for single gene disorders. NIPS also cannot replace imaging by ultrasound studies.

5. The guidelines stress three steps for the introduction of a new test: (i) analytic validity, (ii) clinical validity and (iii) clinical utility. Analytic validity refers to sensitivity (detection rate; DR) and specificity (how many those not having the condition are correctly identified as negative). This has been established for the variety of noninvasive screening methods [14]. Numerous validation studies have been carried out, and they have established that NIPS has very high DR and specificity, reaching nearly 99\% for Down syndrome caused by trisomy 21, translocations, and trisomy 21 mosaicism; $80-100 \%$ for Edwards syndrome caused by trisomy 18 and trisomy 18 mosaicism, as well as for Patau syndrome caused by trisomy 13 , translocations and trisomy 13 mosaicism $[15,16]$. Clinical utility refers to the reliability and usefulness of the test [17]. This is more difficult to establish, and the current methods employ a lot of modeling, and the committee has therefore, not discussed clinical utility of NIPT in their statement.

6. The updated guidelines emphasize the importance of appropriate pre-test genetic counseling, because of the limitations pointed out above. The main objective of the pre-test genetic counseling is to provide the patient information on different methods of prenatal diagnosis, and what each test is capable of diagnosing, in a nondirective way, The benefits and disadvantages of various invasive procedures as well as noninvasive tests should be explained, so that the patients can take an informed decision as to which test is most appropriate for their situation. The statement emphasizes that genetic counseling should not be only educational, but should be patientcentered and take into account the patient's value systems, cultural traditions and religious beliefs. The patient should take a decision once given the necessary facts, alternatives and anticipated consequences. However, in India, many patients with poor knowledge of science have difficulty in comprehending the facts, and point blank ask at the end of the counseling "doctor what you would do if you were in my place". Our response to this is "we cannot be in your place". 
However we do tell them "what most patients with similar reports would do".

7. ACMG also recommends that women at high risk of aneuploidy, either on biochemical tests or on ultrasound findings, should be given the option of diagnostic or invasive testing for detection of chromosomal abnormality, in place of going through the process of NIPS. If the combined test risk is more than 1 in 50, invasive testing would be appropriate, rather than NIPS. It is the authors' practice that in women over 38 years NIPT is recommended rather than biochemical screening, unless there are some contraindications for NIPT, such as advanced gestation beyond the time for termination under Indian law (20 weeks), or patient is from a far-off place making it difficult for the patient to return for a re-test. In such circumstances we recommend diagnostic testing through an invasive procedure. Additional benefits of invasive testing are that abnormalities of all chromosomes are revealed, including translocations and deletions. Microarray studies can also be done on fetal cells when indicated e.g., in the presence of an abnormality on the ultrasound studies, as these reveal a background rate of clinically significant copy number variations (CNVs) to be $1-2 \%$. [18]. Moreover, recent studies have indicated that the risk of miscarriage and other complications of invasive procedures are much lower than ones observed earlier (for amniocentesis 1 in 909, and for chronic villus sampling 1 in 454 [19].

8. ACMG states that the companies offering this test should work with professionals and policymakers to make NIPS accessible to all pregnant women. In India the vendors should register with the PND Act Committee of the area, and get the committee to approve their test, on the assurance that they will not disclose the sex of the fetus in the report, and also will not inform the obstetrician or the geneticist about the sex of the fetus. They should further comply with all the rules and regulations laid down in the Act. The vendors also have a responsibility to provide pre-test and post-test counseling through genetic counselors or obstetricians; although one recognizes that there is a shortage of medical geneticists and genetic counselors in India.

9. One other issue in the Indian setting is that women often report for genetic tests at a later stage in gestation (say more than 19 weeks). In such an event NIPS is not a good option, considering that the report will not be available for $8-10$ days, and the legal limit for termination of pregnancy in India is 20 weeks. Secondly, we refrain from offering NIPS to patients from the peripheral towns where there are no facilities for collection of blood for NIPS testing, or those who come from a great distance which makes it difficult for them to return for a repeat test, in the event of a no call on the test report. To such patients we offer diagnostic testing through an invasive procedure. The NIPT is ideally offered during the first trimester, as there is sufficient time for the report or a repeat sample if it becomes necessary. The obstetricians in India should persuade pregnant women to report in the first trimester so that testing for fetal abnormalities, chromosomal disease or single gene disorders can be carried out in the first trimester, so called reverse pyramid of care promoted by Nicolaides [20].

10. Initially NIPS was offered to only high-risk women. Subsequently experience has been gained in performing NIPS in low-risk women [10,21]. The sensitivity of NIPS is greater than the traditionally used screening options for detecting aneuploidies in low-risk women. Its positive predictive value (PPV) is also higher than the traditional biochemical screening and ultrasound markers. However, the PPV is lower when used in low risk women as compared with its uses in high-risk women. ACMG therefore recommends that all pregnant women be informed that NIPS is the most sensitive screening test for the traditional aneuploidies (trisomy 21,18 , and 13). However, the use of NIPT in low risk women is not accepted by the majority of women in India due to its high cost. Certainly, if the cost of NIPS comes down and NIPS becomes affordable, then it can be offered to women at low risk of aneuploidies, as has a better sensitivity, specificity and positive predictable value. Keeping the high cost of NIPS tests in mind, the most appropriate and affordable protocol in India would be to carry out combined test (dual marker test-PAPP-A and free $\beta$ hCG plus NT), and in those with high risk be offered NIPS, except that in those with very high risk (say more than 1 in 50) invasive testing may be offered.

11. When an increased risk of aneuploidy is reported after NIPS, it is essential that the patient be counseled by a professional with good knowledge of NIPT and its implications, to bring home the point that diagnostic testing is essential. In no case should a woman with a high-risk NIPT be allowed to proceed to termination of pregnancy.

12. If the invasive testing confirms the presence of a chromosomal disease balanced information should be provided at appropriate literacy level, to educate the couple regarding the chromosomal disorder, its prognosis as well as the facilities available for care. However, in the event of diagnosis of an autosomal trisomy, most women in India opt for termination of pregnancy. Only an occasional woman decides to 
continue the pregnancy, especially if she is of advanced maternal age and has failed to conceive for a long time. Counseling in the presence of a sex chromosomal aneuploidy, especially XXY, XXX or $\mathrm{XYY}$, is more difficult as the prognosis is variable. In XXY apart from infertility there may not be other significant problems, while an XYY person may be completely normal, as the violent behavior initially reported in this abnormality was later shown to be a bias of ascertainment in the high security prisons. The mild behavioral problems may be acceptable especially by those women who have an advanced age and who do not have children.

13. ACMG recommends that laboratory reports should clearly state the detection rate, specificity, positive and negative predictive value of their tests. This would assist patients and providers in making decisions and interpreting the results of their tests. This is an important point for vendors to comply with. Patient-specific positive predictive value (PPV) should be provided when reporting positive test results. If patient specific PPV cannot be provided that labs should state population based values, or modeled PPV. If these values are not given the physician should use validated online calculators to provide patient-specific PPV when results from NIPS are positive to facilitate clear and accurate communication with patients.

14. Some vendors provide screening for other chromosomal aneuploidies such as for chromosome 16 or 22. ACMG does not recommend screening for aneuploidies other than trisomy 21,18 , and 13 , because of lack of sufficient validation of testing for these extra autosomes, as well as their clinical value. The frequency of trisomies of these chromosomes would be extremely low in pregnancies beyond the first trimester, and usually end in fetal loss [22].

15. One important issue with NIPT is the frequency of "no call" for the report. This is often due to a low fetal fraction or the test having been performed in a very obese woman, or very early in pregnancy (less than 9 weeks) [23]. It is generally agreed that for an accurate and reliable report the minimum fetal fraction is $4 \%$, and below this amount of fetal fraction the report may be unreliable. Under such circumstances most vendors advise a repeat test. This might be appropriate if the NIPT has been performed early in pregnancy (less than 12 weeks), as there is adequate time for getting the results. However, from 12 to 20 weeks the fetal fraction increases less than $0.1 \%$ per week, which challenges the idea that repeating sample collection is useful in cases of no call due to low fetal fraction [24]. Therefore, ACMG recommends that such women be offered diagnostic testing. In our experience in cases of no call repeat testing has been successful in about $60 \%$ of the cases.

16. As fetal fraction is very critical to obtain an accurate result ACMG recommends that all laboratories should state the fetal fraction in their NIPS reports. One cannot confidently rely on tests that do not state fetal fraction. The instance where two investigators sent blood from nonpregnant women to all the companies offering this test should be remembered [25]. Companies who rely on counting method after massively parallel sequencing reported a female fetus, while the two companies who estimate and report fetal fraction said that the fetal fraction was too low and the tests should be repeated on fresh samples.

17. ACMG recommends that all laboratories should state the reason for a "no-call" in their reports. The professionals and the patients can then better understand why the test failed to provide a result. Two common reasons are a low fetal fraction as stated above or technical reasons. In India and other countries with a high rate of consanguinity there may be long stretches of homozygosity in the DNA and this may be the reason for a no call in tests performed on SNP analysis. In such situations sufficient number of SNPs should be included so that enough remain to provide the necessary information. However whenever stretches of homozygosity are reported the geneticist should look into the possibility of uniparental disomy which may lead to the unmasking of a recessive disorder [26].

18. Some countries offer NIPT screening only for the three aneuploidies, and consider that screening for sex chromosomes is not necessary, as syndromes such as XXY and XYY are compatible with a reasonably good life. In India couples prefer to know abnormalities of the sex chromosomes as they desire the baby should not face "any" problems after birth. On this issue ACMG recommends that all women be informed about the availability of screening for sex chromosome aneuploidies through NIPT and leave the choice to the couple if they would like these to be included in the test protocol. However, it should be kept in mind that the positive predictive value of NIPT for sex chromosomal abnormalities ranges from 38 to $67 \%$, and is lower than that for the autosomal aneuploidies [27]. ACMG emphasizes that sex chromosome screening should not be resorted to for the purpose of knowing the sex of the baby, unless there is a clinical indication for doing so, such as in a sexlinked disorder. Determination of sex is not permitted by law in India except when there is X-linked disease. In India the laboratories screen for sex chromosomes 
but do not report sex of the baby so as to comply with the Indian law. However they do report if there is an abnormality of the sex chromosomes. If the presence of a sex chromosomal disorder is confirmed the couple should be provided accurate and balanced information at an appropriate literacy level, to educate prospective parents about the specific condition.

19. A number of studies have been carried out using NIPS to detect microdeletions. Unfortunately, these tests have not been properly validated due to the lack of availability of sufficient number of patients having microdeletions. Therefore, the validity of these tests is established by spiking the samples with diluted quantities of DNA obtained from cells of patients with microdeletions. However, the positive predictive values of these tests are low. In one large study [28] consisting of about 21,000 women the PPV was only $19 \%$ (varying from 11 to $48 \%$ in different studies). ACMG recommends that all pregnant women be informed about the availability of tests to detect microdeletions, and also be told about the low positive predictive values of these tests, as well as greater false positive and false negative results. Whenever the situation demands copy number variation analysis by using NIPT, the couple should be informed that even more detailed information would be available by study of fetal DNA, obtained through an invasive procedure, using microarrays. If the NIPT detects a microdeletion this must be confirmed by an invasive test. After confirmations, reference to a geneticist is required to provide appropriate counseling regarding the disorder.

20. Screening for CNV over the whole genome is not recommended by ACMG. If this level of information is desired, then diagnostic testing (e.g., chorionic villous sampling or amniocentesis) followed by a microarray study is recommended.

21. With regard to pregnancies with multiple gestations and/or donor oocytes vendors who utilize the counting method after massively parallel sequencing, are successful in providing a report. Tests based on SNPs cannot give an answer under these circumstances. Therefore, one should make sure from the particular vendor that their technology will be able to generate a report. The ACMG feels that more data on twins is required before use of NIPT can be recommended with confidence.

22. Occasionally a bizarre pattern of DNA reads is obtained. Such cases have arisen from the presence of a malignancy in the pregnant woman, the aberrant DNA sequences being derived from the malignant cells [29]. The couple should be informed that occasionally such incidental findings may be obtained. Such cases should be referred to a geneticist for proper counseling who would request the physician to examine and evaluate for the presence of malignancy. Inaccuracies in the NIPT report may be introduced if the pregnant woman is the recipient of a bone marrow transplant or has received blood transfusion within the prior 4 weeks of the test.

23. The physicians should maintain a list of resources for information on common aneuploidies, CNVs or other commonly observed abnormalities, as well as resources that explain in simple language about common terms used in genetics. Such lists should be provided to the patients. It is the authors' practice to hand out an information leaflet about NIPT, its limitations and advantages and the various resources. The couple is advised to read this carefully before choosing the test that they would like to have. In case of an abnormal result appropriate counseling is provided or reference is given to the internet resources. Good resources for patients are Genetic Support Foundation (https://www.geneticsupportfoun dation.org) and National Society of Genetic Counselors (nsgc.org, statements 385 and 387). For professionals the Gene Reviews website (http://www. ncbi.nlm.nih.gov) is recommended.

\section{References}

1. Ehrich M, Deciu C, Zwiefelhofer T, et al. Noninvasive detection of fetal trisomy 21 by sequencing of DNA in maternal blood: a study in a clinical setting. Am J Obstet Gynecol. 2011;204:205.e1-11.

2. Khalifeh A, Weiner S, Berghella V, Donnenfeld A. Trends in invasive prenatal diagnosis: effect of sequential screening and noninvasive prenatal testing. Fetal Diagn Ther. 2016;39:292-6. doi: $10.1159 / 000441028$.

3. Gregg AR, Gross SJ, Best RG, et al. ACMG statement on noninvasive prenatal screening for fetal aneuploidy. Genet Med. 2013;15:395-8.

4. Benn P, Borell A, Chiu R, et al. Position statement from the aneuploidy screening committee on behalf of the board of the international society for prenatal diagnosis. Prenat Diagn. 2013;33(7):622-9.

5. American College of Obstetricians and Gynecologists Committee on Genetics. Committee Opinion No. 545: noninvasive prenatal testing for fetal aneuploidy. Obstet Gynecol. 2012;120(6):1532-4.

6. Wilson KL, Czerwinski JL, Hoskovec JM, et al. NSGC practice guideline: prenatal screening and diagnostic testing options for chromosome aneuploidy. J Genet Couns. 2013;22(1):4-15. doi:10.1007/s10897-012-9545-3 Epub 2012 Nov 22.

7. https://www.smfm.org/publications/157-smfm-statement-mater nal-serum-cell-free-dna-screening-in-low-risk-women.

8. Gregg AR, Skotko BG, Benkendorf JL, Monaghan KG, Bajaj K, Best RG, Klugman S, Watson MS. Noninvasive prenatal screening for fetal aneuploidy, 2016 update: a position statement 
of the American College of Medical Genetics and Genomics. Genet Med. 2016;18(10):1056-65. doi:10.1038/gim.2016.97 (PubMed PMID: 27467454).

9. Dash P, Puri RD, Kotecha U, Bijarnia S, Lall M, Verma IC. Using noninvasive prenatal testing for aneuploidies in a developing country: lessons learnt. J Fetal Med. 2014;1(3):131-5.

10. Zhang H, Gao Y, Jiang F, et al. Non-invasive prenatal testing for trisomies 21,18 and 13: clinical experience from 146,958 pregnancies. Ultrasound Obstet Gynecol. 2015;45:530-8.

11. Hahnemann JM, Vejerslev LO. Accuracy of cytogenetic findings on chorionic villus sampling (CVS)—diagnostic consequences of CVS mosaicism and non-mosaic discrepancy in centres contributing to EUCROMIC 1986-1992. Prenat Diagn. 1997;17(9):801-20.

12. Midtrimester amniocentesis for prenatal diagnosis. Safety and accuracy JAMA. 1976;236(13):1471-6.

13. Gil MM, Quezada MS, Revello R, Akolekar R, Nicolaides KH, et al. Analysis of cell-free DNA in maternal blood in screening for fetal aneuploidies: updated meta-analysis. Ultrasound Obstet Gynecol. 2015;45(3):249-66. doi:10.1002/uog.14791 (Review. PubMed PMID: 25639627).

14. Stokowski R, Wang E, White K, et al. Clinical performance of non-invasive prenatal testing (NIPT) using targeted cell-free DNA analysis in maternal plasma with microarrays or next generation sequencing (NGS) is consistent across multiple controlled clinical studies. Prenat Diagn. 2015;35:1243-6.

15. Palomaki GE, Kloza EM, Lambert-Messerlian GM, et al. DNA sequencing of maternal plasma to detect Down syndrome: an international clinical validation study. Genet Med. 2011;13:913-20.

16. Nicolaides KH, Syngelaki A, Gil M, Atanasova V, Markova D. Validation of targeted sequencing of single-nucleotide polymorphisms for non-invasive prenatal detection of aneuploidy of chromosomes 13, 18, 21, X, and Y. Prenat Diagn. 2013;33:575-9.

17. Kaimal AJ, Norton ME, Kuppermann M. Prenatal testing in the genomic age: clinical outcomes, quality of life, and costs. Obstet Gynecol. 2015;126:737-46.

18. Wapner RJ, Martin CL, Levy B, et al. Chromosomal microarray versus karyotyping for prenatal diagnosis. $\mathrm{N}$ Engl $\mathrm{J}$ Med. 2012;367:2175-84.
19. Akolekar R, Beta J, Picciarelli G, Ogilvie C, D'Antonio F. Procedure-related risk of miscarriage following amniocentesis and chorionic villus sampling: a systematic review and meta-analysis. Ultrasound Obstet Gynecol. 2015;45:16-26.

20. Nicolaides KH. A model for a new pyramid of prenatal care based on the 11 to 13 weeks' assessment. Prenat Diagn. 2011;31:3-6.

21. Norton ME, Jacobsson B, Swamy GK, et al. Cell-free DNA analysis for noninvasive examination of trisomy. N Engl J Med. 2015;372:1589-97.

22. Nussbaum RL, McInnes RR, Willard HF. Thompson \& Thompson Genetics in Medicine. 8th ed. Philadelphia: Elsevier; 2007.

23. Kinnings SL, Geis JA, Almasri E, et al. Factors affecting levels of circulating cell-free fetal DNA in maternal plasma and their implications for noninvasive prenatal testing. Prenat Diagn. 2015;35:816-22.

24. Wang E, Batey A, Struble C, Musci T, Song K, Oliphant A. Gestational age and maternal weight effects on fetal cell-free DNA in maternal plasma. Prenat Diagn. 2013;33:662-6.

25. Takoudes T, Hamar B. Performance of non-invasive prenatal testing when fetal cell-free DNA is absent. Ultrasound Obstet Gynecol. 2015;45:112. doi:10.1002/uog.14715.

26. Rehder CW, David KL, Hirsch B, Toriello HV, Wilson CM, Kearney HM. American College of Medical Genetics and Genomics: standards and guidelines for documenting suspected consanguinity as an incidental finding of genomic testing. Genet Med. 2013;15:150-2.

27. Porreco RP, Garite TJ, Maurel K, et al. Obstetrix Collaborative Research Network. Noninvasive prenatal screening for fetal trisomies $21,18,13$ and the common sex chromosome aneuploidies from maternal blood using massively parallel genomic sequencing of DNA. Am J Obstet Gynecol. 2014;211:365.e1-12.

28. Wapner RJ, Babiarz JE, Levy B, et al. Expanding the scope of noninvasive prenatal testing: detection of fetal microdeletion syndromes. Am J Obstet Gynecol. 2015;212:332.e1-9.

29. Bianchi DW, Chudova D, Sehnert AJ, Bhatt S, Murray K, Prosen TL, Garber JE, Wilkins-Haug L, Vora NL, Warsof S, Goldberg J, Ziainia T, Halks-Miller M. Noninvasive prenatal testing and incidental detection of occult maternal malignancies. JAMA. 2015;314(2):162-9. doi:10.1001/jama.2015.7120. 